\title{
Expression of cluster of differentiation 151 prior to and following transcatheter arterial chemoembolization therapy in patients with hepatocellular carcinoma and its association with clinicopathological characteristics
}

\author{
ZHEN KANG $^{1}$ and ENHUA XIAO ${ }^{2}$ \\ ${ }^{1}$ Department of Radiology, Tongji Hospital, Tongji Medical College, Huazhong University of \\ Science and Technology, Wuhan, Hubei 430030; ${ }^{2}$ Department of Radiology, The Second \\ Xiangya Hospital, Central South University, Changsha, Hunan 410000, P.R. China
}

Received November 14, 2016; Accepted September 13, 2017

DOI: $10.3892 / \mathrm{ol} .2017 .7371$

\begin{abstract}
Cluster of differentiation (CD)151, a member of tetraspanin family, is considered to be the first tetraspanin to be associated with tumor metastasis. Previous studies in vivo, in vitro and in the clinic have demonstrated that CD151 is involved in tumor progression at different levels through interaction with integrins, growth factor receptors and matrix metalloproteinases. Transcatheter arterial chemoembolization (TACE) is widely recommended for the treatment of patients with advanced hepatocellular carcinoma (HCC) worldwide. It has been hypothesized that TACE may create a hypoxic-ischemic environment that increases the expression of tumor progression-associated factors, promotes the angiogenesis of $\mathrm{HCC}$, and initiates the recurrence and metastasis of HCC. Whether TACE promotes HCC progression remains controversial and numerous studies have focused on the influence of TACE on a number of tumor progression-associated factors. In the present study, the expression of serum CD151 in patients with HCC prior to and following TACE and its association with clinicopathological characteristics was investigated. It was revealed that the expression level of CD151 at 5-7 days post-TACE was significantly increased compared with pre-TACE levels. Risk factors and protective factors associated with tumor progression following a single TACE procedure and 18 months of follow-up were also identified. Furthermore, the present study revealed that a pre-TACE CD151 level of $>0.3247 \mathrm{ng} / \mathrm{ml}$ and a 5-7 days post-TACE CD151 level of $>0.3146 \mathrm{ng} / \mathrm{ml}$ revealed moderate sensitivity
\end{abstract}

Correspondence to: Professor Enhua Xiao, Department of Radiology, The Second Xiangya Hospital, Central South University, 139 Renmin Road, Changsha, Hunan 410000, P.R. China

E-mail: xiaoenhua64@csu.edu.cn

Key words: hepatocellular carcinoma, transcatheter arterial chemoembolization, cluster of differentiation 151, tumor progression and specificity for predicting HCC progression following a single TACE procedure. The present study highlights CD151 as a useful marker in predicting the response to treatment and monitoring the disease course following TACE.

\section{Introduction}

Hepatocellular carcinoma (HCC) is the most common primary malignant cancer in the liver, accounting for $\sim 90 \%$ of liver cancer cases and ranking as the fifth most common cancerous disease in males and the ninth in females worldwide in 2012 (1). In total, 30-40\% of patients with HCC in Asia are suitable for potentially curative therapy, including surgical resection and liver transplantation, since patients are often diagnosed at moderate and advanced stages (2).

Transcatheter arterial chemoembolization (TACE) is recommended for the treatment of advanced patients with HCC worldwide. This approach is based on blocking the arteries feeding the tumor, causing irreversible damage (3), necrosis and apoptosis of local tumor cells and endothelial cells in an ischemic anoxia environment and thereby inhibiting the growth of tumor cells, as confirmed using two meta analyses $(4,5)$. For those patients not eligible to receive surgical resection and who are candidates to receive systemic chemotherapy or conservative treatment, TACE serves as an improved, more tolerable option (6). TACE may benefit patients with HCC preoperatively and postoperatively. Preoperative TACE may shrink the tumor volume, reduce the blood supply to the tumor, make surgical excisions easier and embolize small, difficult to visualize tumors (7). Postoperative TACE is used to treat recurrent $\mathrm{HCC}$ and may improve the survival rate and slow the progression of the disease (8).

However, opposing arguments suggest that preoperative TACE may damage liver function, possibly leading to liver failure, and that it may delay surgical resection, make previously resectable liver cancer unresectable and leave residual tumor cells that are more aggressive in cases of poorly-differentiated HCC. Furthermore, incomplete tumor necrosis may reduce the adhesion of tumor cells, allowing them free access 
to the blood, which may result in intrahepatic or extrahepatic metastasis (9). Postoperative TACE may also damage the residual liver function and activate the hepatitis $\mathrm{B}$ virus, which may in turn lead to extrahepatic metastases and a poor prognosis $(10,11)$. Whether TACE promotes progression remains controversial, and a number of studies have focused on the influence of TACE on numerous tumor progression-associated factors, including the expression of vascular endothelial growth factor (VEGF) and hypoxia inducible factor 1- $\alpha$. It has been proposed that TACE may create a hypoxic-ischemic environment that would increase the expression of such factors, promote the angiogenesis of $\mathrm{HCC}$, and initiate the recurrence and metastasis of HCC (12-14).

Cluster of differentiation (CD)151 was the first tetraspanin to be associated with tumour metastasis (15), including gastric, colon, lung and prostate cancer metastasis (16-19), and is believed to be associated with a poor prognosis. A previous study conducting an in vitro experiment demonstrated that cells expressing high levels of CD151 secrete more matrix metalloproteinase (MMP)-9 and possess a marked ability for invasion and metastasis, whilst in the same previous study an in vivo experiment demonstrated that patients expressing high levels of CD151 exhibited a decreased overall survival rate at 3, 5 and 7 years and an increased recurrence rate (15).

The present study aimed to prospectively assess the level of serum CD151 prior to and following TACE, and its association with clinicopathological characteristics.

\section{Materials and methods}

Materials and reagents. The equipment and reagents used in this study included a digital angiography machine (Innova 3100; GE Healthcare, Chicago, IL, USA), a high-pressure injector (Medrad Mark V ProVis; Bayer, Newbury, UK), 2\% lidocaine hydrochloride (Shanghai Zhaohui Pharmaceutical Co., Ltd, Shanghai, China), an 18-G puncture needle (Terumo Medical Corporation, Tokyo, Japan), a 5-F arterial sheath (Terumo Medical Corporation), a 5-F Yashiro catheter (Terumo Medical Corporation), a 5-F RH catheter (Terumo Medical Corporation), an 0.035-inch Loach guide wire (Terumo Medical Corporation), a microtubule (Terumo Medical Corporation), micro guide wire (Terumo Medical Corporation), ioversol(100 ml:67.8 g; Jiangsu Hengrui Medicine Co., Ltd., Lianyungang, China), iodinized oil (1 ml; Shandong Luyin Pharmaceutical Co., Shandong, China), pirarubicin (10 mg; Shenzhen Wanle Pharmaceutical Co. Ltd., Shenzhen, China), fluorouracil (10 ml: 0.25 g; Hainan Zhonghua United Pharmaceutical Industry Co. Ltd., Hainan, China), nedaplatin (10 mg; Aosaikang Pharmaceutical Co. Ltd., Jiangsu, China), a CD151 ELISA detection kit (cat. no. CSB-EL00HU; Cusabio Life Science, Baltimore, MD, USA), a high-speed centrifuge (Thermo Fisher Scientific, Inc., Waltham, MA, USA), a microplate absorbance reader (Thermo Fisher Scientific, Inc.) and a pipettor (Eppendorf, Hamburg, Germany).

Inclusion/exclusion criteria. The inclusion criteria were as follows: Patients with HCC, regardless of primary or recurrent disease or metastasis, with a diagnosis based on clinical symptoms (emaciation, anorexia, abdominal distension, abdominal pain or liver mass, etc.) and imaging [liver mass on computed tomography (CT) or magnetic resonance imaging (MRI)] and a serological $\alpha$-fetoprotein (AFP) level of $>400 \mathrm{ng} / \mathrm{ml}$ or confirmation using pathological analysis. The exclusion criteria consisted of patients with HCC confirmed clinically or pathologically but who rejected TACE.

Serum specimen collection and conservation. The present study was approved by Ethics Committee of the Second Xiangya Hospital (Changsha, China) and written informed consent was collected from each patient who took part in this study. Blood specimens were collected pre-TACE, at 5-7 days post-TACE and at 30 days post-TACE. Samples were centrifuged in a high-speed centrifuge $\left(1,006.2 \mathrm{x} \mathrm{g}\right.$ at $4^{\circ} \mathrm{C}$ for $\left.3 \mathrm{~min}\right)$ and the serum was collected and stored in Eppendorf tubes at $-70^{\circ} \mathrm{C}$.

TACE procedures. Radiologists at the Second Xiangya Hospital performed TACE procedures. Following anesthesia of the right femoral artery sheath with $2 \%$ lidocaine and deposition of a 5-F arterial sheath, a vascular catheter was inserted into the celiac artery. Following conventional hepatic angiography, a vascular catheter was subsequently inserted superselectively into the branch of the hepatic artery that was believed to feed the tumor. Chemoembolization was then performed. Lipiodol (10 ml) was mixed with $40 \mathrm{mg}$ pirarubicin, $80 \mathrm{mg}$ nedaplatin and $1 \mathrm{~g}$ fluorouracil to create an emulsified milky solution. The emulsion was slowly infused into the liver tumor. The dose administered depended on the size of the HCC tumor, the position of the catheter, the patient's liver function, and the patient's tolerance and response to previous courses of TACE. The embolization procedure was abandoned if the patient was unable to tolerate treatment or approached predetermined limits of $15 \mathrm{ml}$ emulsion or the emulsion flow showed stasis or backflow.

ELISA measurement of serum CD151. A detection kit for human serum CD151 was used according to the manufacturer's protocol. The minimum concentration of human CD151 that could be detected was $0.039 \mathrm{ng} / \mathrm{ml}$. Optical density (OD) values were transformed to the concentration of serum CD151. Standard curves were calculated using the standard provided with the kit. The standard specimen information is presented in Table I. Based on the OD values measured using ELISA and the corresponding concentration of CD151, a scatter diagram was created and the following equation/values were obtained: $\mathrm{y}=0.1406 \mathrm{e}^{5.1986 \mathrm{x}}, \mathrm{R}^{2}=0.9514$ (Fig. 1).

Statistical analysis. SPSS (version 17.0; SPSS, Inc., Chicago, IL, USA), MedCalc (version 11.4.2.0; MedCalc Software bvba, Ostend, Belgium) and GraphPad Prism (version 5.0; GraphPad Software, Inc., La Jolla, CA, USA) software were used for the statistical analysis. Descriptive statistics of the serum CD151 level were used to calculate the means and ranges of the distribution (range and standard deviation). Mann Whitney test was used to compare differences between two independent samples. Paired Student's t-tests were used to compare differences between the serum CD151 values pre- and post-TACE. Correlation analysis was used to evaluate the correlation of measurement data. Kaplan-Meier estimator analysis was used to generate the survival curve. Single-factor and multi-factor 
Table I. Information for the standard specimen.

\begin{tabular}{lllllll}
\hline Serial & S5 & S4 & S3 & S2 & S1 & S0 \\
\hline Absorbance (blank removed) & 0.5957 & 0.3550 & 0.2746 & 0.1408 & 0.0681 & 0.0000 \\
Concentration, ng/ml & 2.500 & 1.250 & 0.625 & 0.312 & 0.156 & 0.000 \\
\hline
\end{tabular}

A stock solution was used to produce a 2-fold dilution series (S5-S1). Sample diluent served as the zero standard (0 ng/ml).

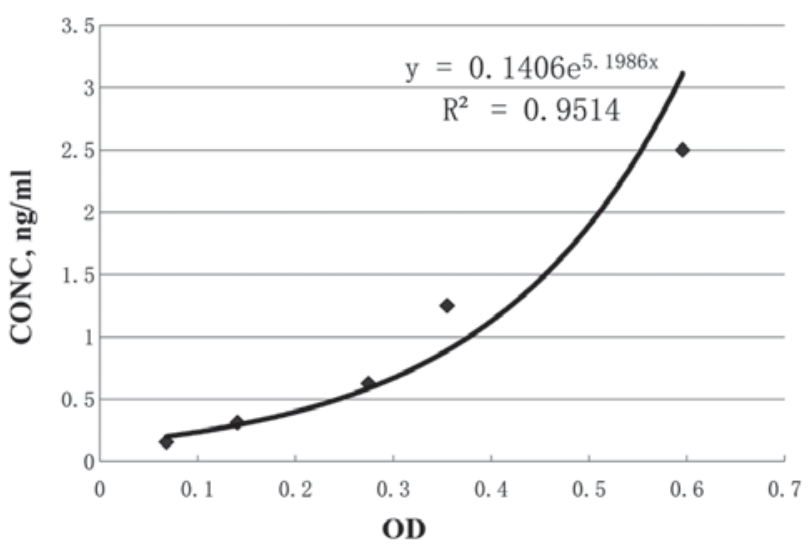

Figure 1. Scatter diagram of the standard curve and equation for cluster of differentiation 151. OD, optical density; CONC, concentration.

logistic regression analyses were used to assess the risk factors associated with the progression of HCC. Receiver operating characteristic (ROC) curves were generated to assess CD151 expression in predicting the progression of HCC. Threshold values for neutrophil to lymphocyte ratio (NLR), platelet to lymphocyte ratio (PLR) and platelet count were based on previous studies (20-22). A two-tailed P-value of $<0.05$ was considered to indicate a statistically significant difference.

\section{Results}

Clinical data of the included patients. Patients with HCC $(n=28)$ were selected, including 26 males and 2 females. A total of 21 patients had not previously received TACE, while 7 patients had previously received TACE. The majority of patients were diagnosed according to their clinical symptoms, together with imaging results and serum AFP levels (>400 ng/ml), while the 7 remaining patients were diagnosed using pathological analysis through CT-guided biopsy due to serum AFP levels being below the aforementioned threshold. The mean average age of these patients was $49.8 \pm 17.7$ years (range, 22-72 years). Hepatitis B surface antigen (HBsAg) positivity was identified in $23 / 28$ patients. Of all the selected patients, 24 were classified as Child-Pugh A and 4 as Child-Pugh B (23). The tumor mass measured $8.5-13 \mathrm{~cm}$ in the treatment-naïve patients. Portal thrombus was identified in 10 patients. A total of 9 patients had recurrent or metastatic HCC presenting with multiple nodules upon imaging. The 7 patients who had received TACE previously exhibited poor iodinized oil deposition and required intensive therapy. The baseline clinical data are presented in Table II.
Expression of serum CD151 in patients with HCC treated by TACE

Serum CD151 expression pre-TACE and the association with the baseline clinical data. The expression of CD151 pre-TACE and the association with the baseline clinical data were analyzed. The Mann Whitney test revealed that the expression of CD151 pre-TACE demonstrated no statistically significant association with the patient's age, sex, HBsAg status, vascular invasion status, AFP level, HCC type, NLR, PLR, platelet count or TACE number received, as presented in Table III.

Serum CD151 expression 5-7 days post-TACE and the association with the baseline clinical data. The expression levels of CD151 5-7 days post-TACE and the association with the baseline clinical data were analyzed, and the Mann Whitney test revealed that the expression of CD151 5-7 days post-TACE was significantly increased in recurrent or metastatic HCC compared with primary HCC $(\mathrm{P}=0.004$; Table III).

Comparison of CD151 expression pre-TACE and at 5-7 days post-TACE. The mean average CD151 expression level pre-TACE was $0.35 \mathrm{ng} / \mathrm{ml}$, compared with $0.46 \mathrm{ng} / \mathrm{ml}$ at 5-7 days post-TACE, with the paired t-test revealing a statistically significant difference between time points $(\mathrm{P}<0.001$; Table IV; Fig. 2).

Comparison of CD151 expression pre-TACE and at 30 days post-TACE. Serum specimens from 12 patients with HCC who were not previously treated were obtained at 30 days post-TACE. A paired student's t-test revealed no significant difference in the expression of CD151 pre-TACE and at 30 days post-TACE (Table V; Fig. 3).

CD151 expression pre-and post-TACE. Among the 12 patients who provided serum samples for all three time points, the mean average levels of CD151 expression pre-TACE, at 5-7 days post-TACE and at 30 days post-TACE were $0.35,0.41$ and $0.42 \mathrm{ng} / \mathrm{ml}$, respectively. These results identified a tendency for a rapid increase followed by a relatively slower increase, although no statistically significant differences were identified ( $\mathrm{P}=0.44$; Fig. 4).

Follow-up standard. Imaging and digital subtraction angiography findings for patients who received a second TACE treatment were reviewed, and the Response Evaluation Criteria for Solid Tumors (24) were modified and applied for assessment of the progression of HCC. During imaging analysis, tumor progression was reported if the largest diameter of the mass had increased since the previous examination or if a new 
Table II. Baseline clinical data of the included patients.

Characteristic

\section{Sex}

Male

Female

HBsAg

$+$

$-$

TACE received prior to study

Treatment naïve

$>1$ session

Child-Pugh stage

A
B
C

Vascular invasion

Portal thrombus

No vascular invasion

AFP level, ng/ml

$>400$

$<400$

HCC type

Recurrent or metastatic HCC Primary HCC

HBsAg, hepatitis B surface antigen; TACE, transcatheter arterial chemoembolization; AFP, $\alpha$-fetoprotein; HCC, hepatocellular carcinoma.

mass or nodule was noted beside the previous mass (Fig. 5). On the digital subtraction angiograph, tumor progression was considered if the feeding blood vessels had increased in size or vascularity and were disordered. However, if the mass did not exhibit any marked change in size or vascularity, the tumor was not considered to have progressed and remained under control (Fig. 6).

Follow-up data. During the 18-month follow-up period, $9 / 28$ patients were lost to follow-up and 2 outpatients exhibited tumor progression and did not receive additional treatment. A total of 11 patients were regarded as censored. The remaining 17 patients received the second TACE treatment within the first 2 months. At the 7-month interval, 12 patients had survived and received another TACE treatment. By the 1-year interval, 9 patients had survived. At the end of 18 months, 5 patients remained (Table VI). Using these data, the approximate survival rate was calculated, revealing a decrease in overall survival rate with time (Fig. 7).

Risk factors associated with the progression of HCC for patients receiving their first TACE. Following the first TACE treatment, 9 patients were censored and classed as exhibiting tumor progression. For the remaining 19 patients, their medical records were reviewed, along with angiography and
CT or MRI images. A total of 9 patients demonstrated marked tumor progression, while the other 10 patients exhibited no tumor progression. In total, there were 18 patients with tumor progression and 10 patients with no tumor progression. Single-factor analysis identified that preoperative high levels of AFP (>400 ng/ml), CD151 expression pre-TACE and CD151 expression 5-7 days post-TACE were risk factors for tumor progression following a single TACE treatment. Embolism volume was a protective factor. Multiple factor analysis failed to provide significant results (Table VII).

The predictive value of CD151 expression pre-TACE and 5-7 days post-TACE for tumor progression was analyzed. ROC analysis revealed that for a CD151 expression level of $>0.3247 \mathrm{ng} / \mathrm{ml}$ pre-TACE, the sensitivity and specificity to predict tumor progression were 66.7 and $80.0 \%$, respectively, with an area under the curve (AUC) of 0.753 (95\% confidence interval (CI), 0.554-0.895; $\mathrm{P}=0.007)$. For a CD151 expression level of $>0.3146 \mathrm{ng} / \mathrm{ml}$ at $5-7$ days post-TACE, the sensitivity and specificity to predict tumor progression were 94.4 and $50.0 \%$, respectively, with an AUC of 0.761 (95\% CI, 0.563-0.901; $\mathrm{P}=0.007$ ) (Fig. 8).

Risk factors associated with the progression of HCC for patients at the end of follow-up. The patients were followed up for 18 months. A total of 5 patients exhibited no marked tumor progression, while 14 patients presented with hepatic masses that grew larger or exhibited blood vessels of increased size or vascularity on digital subtraction angiography. In total, 23 patients were deemed to have experienced tumor progression, and 5 patients were deemed to have experienced no tumor progression. Single-factor analysis identified that HBsAg positivity and an NLR of $>1.85$ at the end of follow-up were risk factors for tumor progression among patients receiving TACE. Furthermore, the total number of TACE treatments received and the embolism volume were protective factors (Table VIII). Multiple factor analysis failed to identify any statistically significant differences.

\section{Discussion}

CD151 was first identified on the surface of platelets by Ashman et al (25) in 1991 and was referred to as platelet-endothelial tetraspan antigen-3, the activation of which led to platelet aggregation. Later, cDNA for CD151 was isolated from the meganucleus metrocyte leukemia MO7e cell line and the adult T lymphocytic leukemia SF-HT cell line. In 1997, fluorescence in situ hybridization located the gene for CD151 on chromosome 11p15.5, and gene and protein analysis identified CD151 as part of the tetraspanin family $(26,27)$. This molecule was nominated to join the CD molecular family at the 6th International Human Leucocyte Differentiation Antigen conference (28) and was designated CD151. CD151 is widely expressed in human tissues, including the myocardium, smooth muscle, skeletal muscle cells, endothelial cells, and immune-related Schwann cells and dendritic cells (29). CD151 is now considered an important molecule that is involved in tumor progression. A previous study conducting in vivo, in vitro and clinical experiments has demonstrated that CD151 is involved in tumor progression at different levels (15); CD151 may impact the behaviour of tumor cells, assist tumor cells 
Table III. Association between CD151 expression pre-TACE and at 5-7 days post-TACE and the baseline clinical data.

\begin{tabular}{|c|c|c|c|c|}
\hline Variants & $\begin{array}{l}\text { CD151 pre- } \\
\text { TACE, ng/ml }\end{array}$ & $\begin{array}{c}\text { P-value } \\
\text { (Mann-Whitney test) }\end{array}$ & $\begin{array}{l}\text { CD151 5-7 days } \\
\text { post-TACE, } \mathrm{ng} / \mathrm{ml}\end{array}$ & $\begin{array}{c}\text { P-value } \\
\text { (Mann-Whitney test) }\end{array}$ \\
\hline Age, years & & 0.71 & & 0.8 \\
\hline$\geq 50(n=14)$ & $0.34 \pm 0.08$ & & $0.46 \pm 0.17$ & \\
\hline$\leq 50(n=14)$ & $0.37 \pm 0.11$ & & $0.45 \pm 0.12$ & \\
\hline Sex & & 0.05 & & \\
\hline Male $(n=26)$ & $0.34 \pm 0.1$ & & $0.45 \pm 0.15$ & 0.18 \\
\hline Female $(n=2)$ & $0.49 \pm 0.04$ & & $0.58 \pm 0.08$ & \\
\hline $\mathrm{HBsAg}$ & & 0.06 & & 0.37 \\
\hline$+(n=23)$ & $0.37 \pm 0.1$ & & $0.45 \pm 0.16$ & \\
\hline$-(n=5)$ & $0.29 \pm 0.03$ & & $0.49 \pm 0.1$ & \\
\hline Vascular invasion & & 0.06 & & 0.28 \\
\hline Portal thrombus $(n=10)$ & $0.4 \pm 0.1$ & & $0.51 \pm 0.19$ & \\
\hline No vascular invasion $(\mathrm{n}=18)$ & $0.33 \pm 0.07$ & & $0.42 \pm 0.11$ & \\
\hline AFP, ng/ml & & 0.56 & & 0.06 \\
\hline$>400(n=21)$ & $0.36 \pm 0.11$ & & $0.42 \pm 0.13$ & \\
\hline$<400(\mathrm{n}=7)$ & $0.33 \pm 0.06$ & & $0.56 \pm 0.17$ & \\
\hline HCC type & & 0.09 & & $0.004^{\mathrm{a}}$ \\
\hline Primary HCC $(n=19)$ & $0.33 \pm 0.08$ & & $0.41 \pm 0.15$ & \\
\hline Recurrent or metastatic HCC (n=9) & $0.4 \pm 0.11$ & & $0.55 \pm 0.09$ & \\
\hline NLR & & 0.77 & & 0.87 \\
\hline$<1.85(\mathrm{n}=7)$ & $0.33 \pm 0.02$ & & $0.48 \pm 0.21$ & \\
\hline$>1.85(\mathrm{n}=21)$ & $0.36 \pm 0.11$ & & $0.45 \pm 0.13$ & \\
\hline PLR & & 0.72 & & 0.77 \\
\hline$<111.23(\mathrm{n}=4)$ & $0.35 \pm 0.06$ & & $0.52 \pm 0.28$ & \\
\hline$>111.23(\mathrm{n}=24)$ & $0.36 \pm 0.1$ & & $0.44 \pm 0.12$ & \\
\hline Platelet count & & 0.69 & & 0.95 \\
\hline$<118(\mathrm{n}=4)$ & $0.35 \pm 0.06$ & & $0.52 \pm 0.28$ & \\
\hline$>212(n=13)$ & $0.35 \pm 0.1$ & & $0.46 \pm 0.1$ & \\
\hline TACE received & & 0.20 & & 0.79 \\
\hline Treatment-naïve $(n=21)$ & $0.34 \pm 0.1$ & & $0.46 \pm 0.15$ & \\
\hline$>1(n=7)$ & $0.4 \pm 0.11$ & & $0.46 \pm 0.14$ & \\
\hline
\end{tabular}

The NLR threshold of 1.85 was established according to a previous study (20). The PLR threshold of 111.23 was established according to a previous study (21). The platelet threshold of $118,000 / \mu 1$ or $212,000 / \mu 1$ was also established according to a previous study $(22)$. ${ }^{a}<<0.05$. $C D$, cluster of differentiation; TACE, transcatheter arterial chemoembolization; HBsAg, hepatitis B surface antigen; AFP, $\alpha$-fetoprotein; HCC, hepatocellular carcinoma; NLR, neutrophil to lymphocyte ratio; PLR, platelet to lymphocyte ratio.

in communicating with neighbouring cells and the microenvironment, and regulate the events following tumor adhesion, including cell proliferation, migration and invasion, and the subsequent processes of infiltration and metastasis. These molecular mechanisms involved in cancer processes are based on the ability of CD151 to interact with integrins ( $\alpha 3 \beta 1, \alpha 6 \beta 1$ and $\alpha 6 \beta 4$ ), growth factor receptors (hepatocyte growth factor receptor, epidermal growth factor receptor and transforming growth factor- $\beta 1$ receptor) and MMPs (MMP-7, MMP-2 and MMP-9), which together are involved in the development of cancer (30).

The present study demonstrated that pre-TACE, CD151 expression had no statistical correlation with age, sex, hepatitis

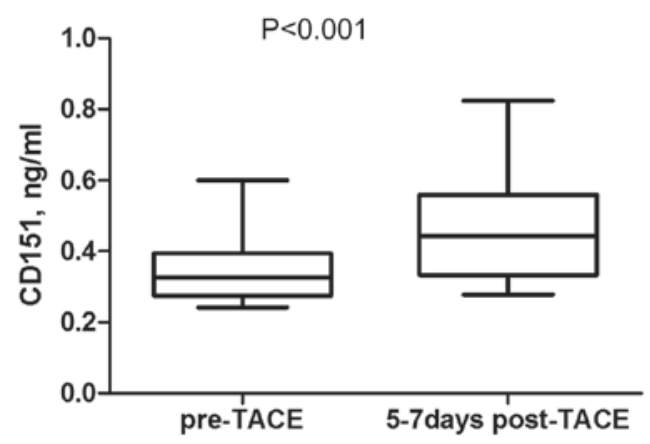

Figure 2. Comparison of CD151 pre-TACE and at 5-7 days post-TACE. CD, cluster of differentiation; TACE, transcatheter arterial chemoembolization. 
Table IV. Comparison of CD151 pre-TACE and at 5-7 days post-TACE.

\begin{tabular}{lcc}
\hline Time & $\begin{array}{c}\text { CD151, } \\
\text { ng/ml }\end{array}$ & $\begin{array}{c}\text { P-value } \\
\text { (two-tailed test) }\end{array}$ \\
\hline Pre-TACE $(\mathrm{n}=28)$ & $0.35 \pm 0.1$ & \\
5-7 days post-TACE $(\mathrm{n}=28)$ & $0.46 \pm 0.15$ & $\mathrm{P}<0.001$ \\
\hline
\end{tabular}

$\mathrm{CD}$, cluster of differentiation; TACE, transcatheter arterial chemoembolization.

Table V. Comparison of CD151 pre-TACE and at 30 days post-TACE.

\begin{tabular}{lcc}
\hline Time & $\begin{array}{c}\text { CD151, } \\
\text { ng/ml }\end{array}$ & $\begin{array}{c}\text { P-value } \\
\text { (two-tailed test) }\end{array}$ \\
\hline Pre-TACE $(\mathrm{n}=12)$ & $0.35 \pm 0.1$ & \\
30 days post-TACE $(\mathrm{n}=12)$ & $0.42 \pm 0.2$ & $\mathrm{P}=0.09$ \\
\hline
\end{tabular}

$\mathrm{CD}$, cluster of differentiation; TACE, transcatheter arterial chemoembolization.

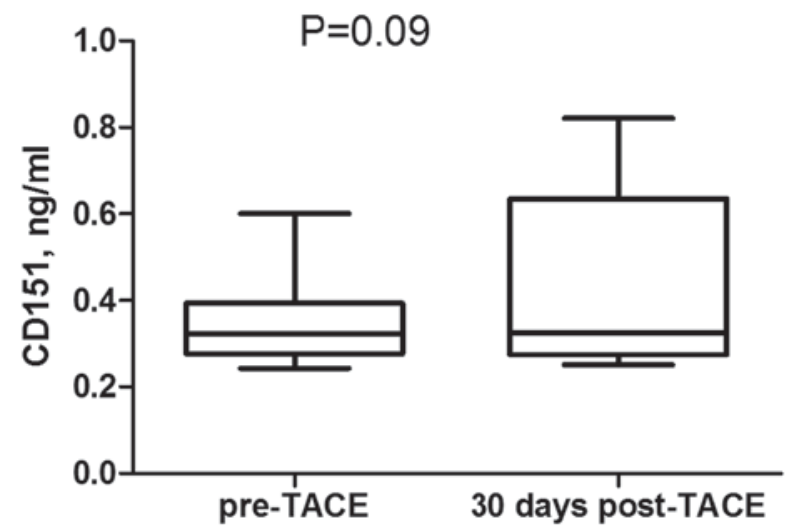

Figure 3. Comparison of CD151 expression pre-TACE and at 30 days post-TACE. CD, cluster of differentiation; TACE, transcatheter arterial chemoembolization.

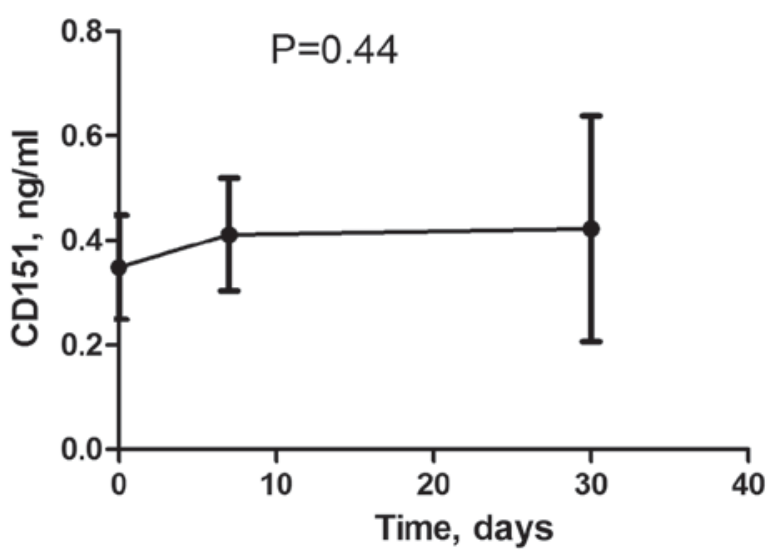

Figure 4. Tendency of CD151 expression pre-TACE and post-TACE. CD, cluster of differentiation; TACE, transcatheter arterial chemoembolization.
Table VI. Follow-up data for the included patients by time interval.

\begin{tabular}{lccc}
\hline $\begin{array}{l}\text { Follow-up } \\
\text { time, months }\end{array}$ & $\begin{array}{c}\text { Patients } \\
\text { remaining }\end{array}$ & $\begin{array}{c}\text { TACE } \\
\text { treatments }\end{array}$ & $\begin{array}{c}\text { Survival } \\
\text { rate, \% }\end{array}$ \\
\hline 0 & 28 & 1 & 100.0 \\
2 & 17 & 2 & 60.7 \\
7 & 12 & $\geq 2$ & 42.9 \\
12 & 9 & $\geq 3$ & 32.1 \\
15 & 7 & $\geq 3$ & 25.0 \\
18 & 5 & $3-5$ & 17.9 \\
\hline
\end{tabular}

TACE, transcatheter arterial chemoembolization.
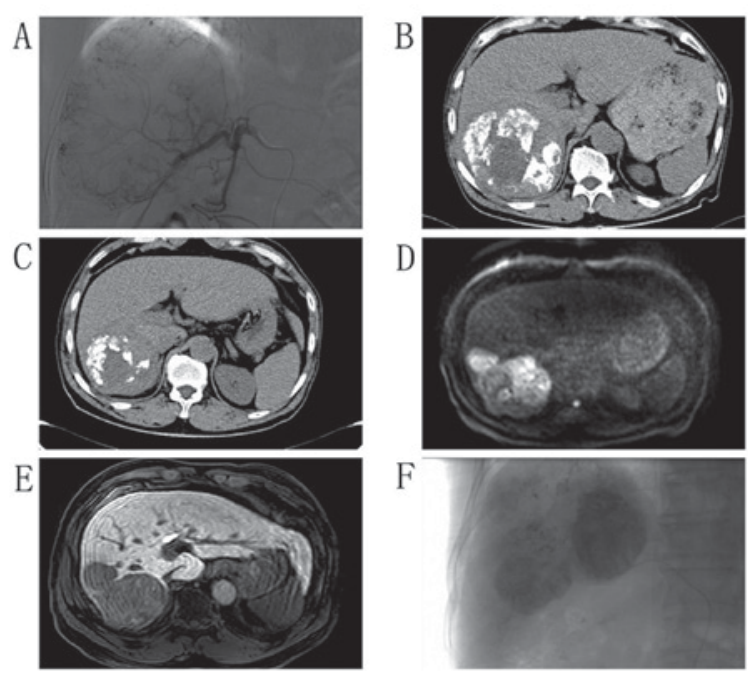

Figure 5. Representative images of a 59-year-old male treatment-naïve patient with hepatocellular carcinoma. (A) Angiography of the tumor revealed rich vascularity, therefore TACE was performed. (B) At 1 month post-TACE, computed tomography examination revealed moderate focal iodine oil deposits, thus the patient received a second TACE treatment. (C) After 4 months, the tumor size exhibited no marked change. (D) The patient received a third TACE treatment and diffusion weighted imaging revealed a new developed nodule with abnormal restricted diffusion, suggesting progression, 1 year later. (E) Gd-EOB-DTPA-enhanced MRI $30 \mathrm{~min}$ after intravenous injection of Gd-EOB-DTPA revealed a new hypo-intensity nodule beside the previous mass. (F) Digital subtraction angiography confirmed rich blood vessels. This case was considered as tumor progression. TACE, transcatheter arterial chemoembolization; MRI, magnetic resonance imaging; Gd-EOB-DTPA, gadolinium ethoxybenzyl diethylenetriamine pentaacetic acid.

B status, vascular invasion, AFP, HCC type, TACE number received, NLR, PLR or platelet count. Post-TACE, CD151 expression in recurrent or metastatic liver cancer cases was significantly more increased compared with the increase observed in primary HCC cases, suggesting that the CD151 expression level is associated with distinct types of HCC following TACE treatment. The results of the present study suggested that CD151 is involved in the metastasis of HCC, which is consistent with the results of the study by Ke et al (15). CD151 expression tended to exhibit a rapid increase within 5-7 days post-TACE, followed by a smaller increase between 5-7 and 30 days post-TACE. The rapid early increase in CD151 
Table VII. Risk factor associations with tumor progression after the first TACE, as assessed by logistic regression analysis.

\begin{tabular}{|c|c|c|c|c|}
\hline \multirow[b]{2}{*}{ Variable } & \multicolumn{2}{|c|}{ Single-factor analysis } & \multicolumn{2}{|c|}{ Multiple factor analysis } \\
\hline & Test statistic & P-value & Test statistic & P-value \\
\hline \multicolumn{5}{|l|}{ Categorical } \\
\hline HBsAg & $1.564^{\mathrm{a}}$ & 0.315 & \multirow{7}{*}{0.000} & \multirow{7}{*}{0.998} \\
\hline Vascular invasion & $0.221^{\mathrm{a}}$ & 0.703 & & \\
\hline AFP & $5.185^{\mathrm{a}}$ & $0.03^{\mathrm{b}}$ & & \\
\hline HCC type & $1.052^{\mathrm{a}}$ & 0.417 & & \\
\hline TACE received & $1.052^{\mathrm{a}}$ & 0.417 & & \\
\hline NLR & $5.185^{\mathrm{a}}$ & 0.063 & & \\
\hline PLR & $0.415^{\mathrm{a}}$ & 0.601 & & \\
\hline \multicolumn{5}{|l|}{ Continuous } \\
\hline CD151 expression pre-TACE & $-2.891^{\mathrm{c}}$ & $0.008^{\mathrm{b}}$ & 2.915 & 0.088 \\
\hline CD151 expression at 5-7 days post-TACE & $-2.353^{\mathrm{c}}$ & $0.026^{\mathrm{b}}$ & 2.760 & 0.097 \\
\hline Embolism volume & $2.999^{c}$ & $0.006^{\mathrm{b}}$ & 2.696 & 0.101 \\
\hline
\end{tabular}

${ }^{\mathrm{a}} \chi^{2}$ test; ${ }^{\mathrm{b}} \mathrm{P}<0.05 ;{ }^{\mathrm{c} t}$-test. $\mathrm{CD}$, cluster of differentiation; TACE, transcatheter arterial chemoembolization; HBsAg, hepatitis B surface antigen; AFP, $\alpha$-fetoprotein; HCC, hepatocellular carcinoma; NLR, neutrophil to lymphocyte ratio; PLR, platelet to lymphocyte ratio.

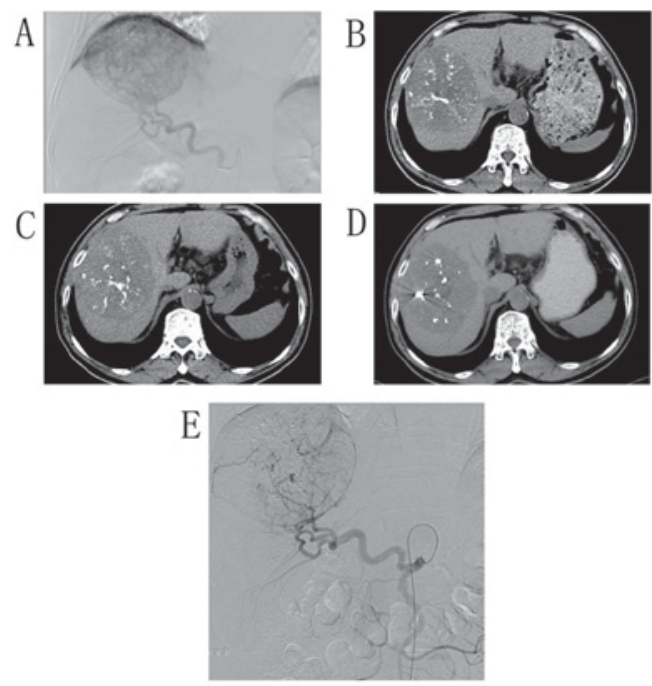

Figure 6. Representative images of a 69-year-old male patient with hepatocellular carcinoma who had received TACE treatment previously. (A) Digital subtraction angiography revealed a well-margined mass in the liver with a clear feeding blood vessel. (B) Following the fifth TACE, computed tomography analysis revealed poor deposition of iodine and the patient received a sixth TACE treatment. (C) 3 months and (D) 1 year after the sixth TACE, the mass size remained similar with (E) no increase in the quantity of blood vessels. This case was considered not to exhibit any tumor progression, although the iodine deposition was poor. TACE, transcatheter arterial chemoembolization.

may indicate the invasive characteristics of tumor cells due to the ischemic anoxic environment.

Univariate analysis identified that factors associated with tumor progression following a single TACE treatment included AFP, pre-TACE CD151 and post-TACE CD151. A previous study demonstrated that AFP may promote AKT phosphorylation through interaction with the phosphatase and

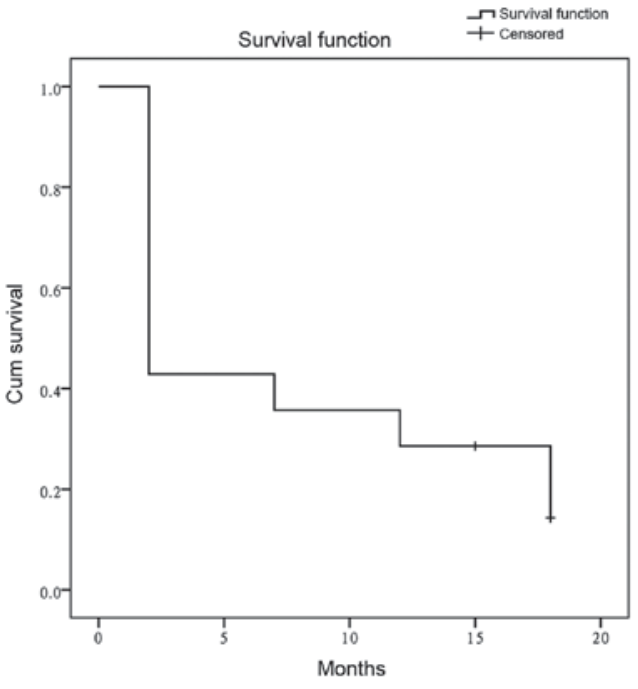

Figure 7. Approximate survival function calculation for the follow-up data.

tensin homolog protein, thereby increasing the expression of the $\mathrm{C}-\mathrm{X}-\mathrm{C}$ chemokine receptor type 4 (CXCR4) protein (also known as stromal-derived factor- $1 \alpha$ ) (31). At the same time, mechanistic target of rapamycin enters the nucleus to combine with the CXCR4 gene promoter, which promotes the growth and metastasis of HCC (28). CD151 was the first tetraspanin to be associated with tumor metastasis. The present study identified pre-TACE CD151 and post-TACE CD151 expression as risk factors for $\mathrm{HCC}$ progression following a single TACE treatment. The results also suggested that interactions may occur between CD151 and AFP. CD151 is able to upregulate MMP-9 through the phosphoinositide 3-kinase/Akt/glycogen synthase kinase-3 $\beta$ /snail signaling pathways (32) and form functional complexes with integrins $\alpha 3 \beta 1$ and $\alpha 6 \beta 1$ to 
Table VIII. Risk factors correlating with tumour progression at the end of follow-up, as assessed by logistic regression analysis.

\begin{tabular}{|c|c|c|c|c|}
\hline \multirow[b]{2}{*}{ Variable } & \multicolumn{2}{|c|}{ Single-factor analysis } & \multicolumn{2}{|c|}{ Multiple factor analysis } \\
\hline & Test statistic & P-value & Test statistic & P-value \\
\hline \multicolumn{5}{|l|}{ Categorical } \\
\hline HBsAg & $7.370^{\mathrm{a}}$ & $0.027^{\mathrm{b}}$ & 0 & 0.998 \\
\hline Vascular invasion & $3.382^{\mathrm{a}}$ & 0.128 & & \\
\hline AFP & $2.029^{\mathrm{a}}$ & 0.290 & & \\
\hline HCC type & $2.883^{\mathrm{a}}$ & 0.144 & & \\
\hline NLR & $18.261^{\mathrm{a}}$ & $<0.001^{\mathrm{b}}$ & 0 & 0.997 \\
\hline PLR & $3.287^{\mathrm{a}}$ & 0.135 & & \\
\hline \multicolumn{5}{|l|}{ Ordinal } \\
\hline Total TACE received & $7^{\mathrm{c}}$ & $0.002^{\mathrm{b}}$ & 0 & 0.996 \\
\hline \multicolumn{5}{|l|}{ Continuous } \\
\hline CD151 expression pre-TACE & $-1.228^{\mathrm{d}}$ & 0.230 & & \\
\hline CD151 expression at 5-7 days post-TACE & $-1.473^{\mathrm{d}}$ & 0.153 & & \\
\hline Embolism volume & $3.824^{\mathrm{d}}$ & $0.001^{\mathrm{b}}$ & 0 & 0.996 \\
\hline
\end{tabular}

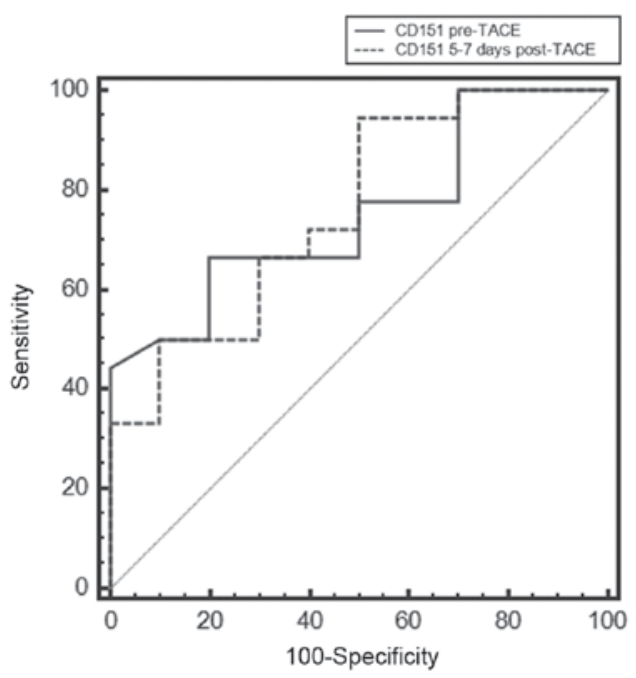

Figure 8. Receiver operating characteristic curve of CD151 expression pre-TACE and at 5-7 days post-TACE for the prediction of tumor progression. TACE, transcatheter arterial chemoembolization; $\mathrm{CD}$, cluster of differentiation.

promote tumor invasion and metastasis $(33,34)$. However, no studies have focused on CD151 and AFP complex formation or signal interaction, which may serve as a promising focus of future research, particularly the genes and underlying molecular mechanisms involved. It has been demonstrated that VEGF expression increases with tumor angiogenesis, and a VEGF-A level of $>16.7 \mathrm{pg} / \mathrm{ml}$ at 7 days post-TACE may serve as an independent criteria for the diagnosis of tumor growth, with a diagnostic sensitivity of $76 \%$ and specificity of $66.7 \%$ (12), which may increase the risk of HCC metastasis (35). The present study revealed that preoperative and 5-7 days postoperative CD151 expression levels were risk factors for HCC progression, and it was identified that a preoperative CD151 level of $>0.3247 \mathrm{ng} / \mathrm{ml}$ and a postoperative CD151 level of $>0.3146 \mathrm{ng} / \mathrm{ml}$ were sufficient to predict HCC progression with a sensitivity and specificity of 66.7 and $80 \%$, and 94.4 and $80 \%$, respectively. Furthermore, the AUC values were 0.753 and 0.761 , respectively, revealing that the detection of CD151 may be used to predict the progression of HCC following TACE, with the diagnostic value of pre- and post-TACE almost equivalent to each other. In the patients with HCC exhibiting tumor progression, it was identified that CD151 expression levels at 5-7 days post-TACE were increased compared with pre-TACE levels; tumor progression slowed following a decrease in CD151 levels, and if CD151 fell below the pre-TACE level, the patient was likely to remain free of tumor progression. If the postoperative CD151 level decreased, the tumor was likely to maintain the same status as assigned previously, indicating that CD151 may serve an important role in the development of liver cancer following TACE. The expression of CD151 in comparison to the preoperative level may be used to predict tumor progression and monitor the effectiveness of TACE treatment.

Embolism volume was identified as a protective factor following a single TACE treatment and at the end of follow-up, regardless of the treatment the patient received. This finding indicates that intervention by physicians should maximize the embolism volume, which may be beneficial to patients with HCC. With an increase in the number of TACE treatments received, $\mathrm{HCC}$ final progression decreased, indicating that TACE did not promote invasion and metastasis of HCC.

The NLR is used as an indicator of the systemic inflammatory response and associates with the prognosis of a number 
of tumors. Patients with a normal NLR post-TACE experience an increased survival time compared with those with an elevated NLR (median survival time, 17.5 and 8 months respectively). Furthermore, the 1-, 3- and 5-year survival rates of patients were improved in those with a normal NLR post-TACE. Previous ROC curve analysis demonstrated that when an NLR of 1.85 was used as the threshold, the NLR pre-TACE predicted the outcome of TACE treatment (20). The present study confirmed this result, identifying that the NLR was one of the risk factors for the final progression of HCC.

Increased platelets are associated with the metastasis of a number of tumors. However, taking into account the development of liver cirrhosis, which is a disease process accompanied by a reduction in platelets, it is unclear whether the platelet count pre-TACE has any association with the patient's outcome. It has been demonstrated that patients with platelet counts of $<118,000 / \mu 1$ exhibit a lower metastatic rate, while a platelet count of $>212,000 / \mu 1$ is associated with tumor metastasis (22). Furthermore, a lower PLR $(\leq 111.23)$ is likely to result in an improved survival rate; the overall survival rate at 3 months was previously reported at $57.6(\leq 111.23)$ vs. $37.6 \%$ ( $\geq 111.23$ ) (21). However, in the present study, no association between the platelet count and PLR and the expression of CD151 and tumor progression was identified, suggesting that platelets serve a weak role in the progression of HCC.

All patients included in the current study presented with large tumors (diameter between 8.5 and $13 \mathrm{~cm}$ ) or recurrent $\mathrm{HCC}$, which is likely to result in a poor prognosis. However, the patients were classified as Child-Pugh A and B, indicating relatively good liver function. According to the Barcelona Clinic Liver Cancer staging system (36), TACE treatment was recommended. Intention analysis was applied in the present study; patients who did not come to the hospital or who were not re-examined were regarded as deceased. The results indicated that with increasing time and TACE treatments, the mortality rate increased; however, the 1-year survival rate was $\sim 32 \%$, indicating satisfactory results of the treatment.

The present study was limited by the small sample size and differences between patients in disease characteristics. Additionally, the majority of the hepatic masses were large and a number of patients were lost to follow-up, which may have had a negative influence on the results. Furthermore, numerous factors may interact with one another during disease progression. It is also hypothesized that serum stored for 1 month may exhibit decreased expression of CD151, even if stored at $-70^{\circ} \mathrm{C}$. Measurement error for ELISA may also exist in the experimental process.

To conclude, the present study identified that CD151 expression at 5-7 days post-TACE was increased compared with that observed pre-TACE, that CD151 expression pre-TACE and at 5-7 days post-TACE served as a risk factor for the progression of HCC, and that the CD151 level may be used to predict the progression of $\mathrm{HCC}$, with optimal threshold values of $>0.3247 \mathrm{ng} / \mathrm{ml} \mathrm{CD151}$ pre-TACE and $>0.3146 \mathrm{ng} / \mathrm{ml}$ at 5-7 days post-TACE. The risk factors for $\mathrm{HCC}$ progression following a single TACE treatment included AFP, pre-TACE CD151 and post-TACE CD151, while the embolism volume served as a protective factor. The risk factors for final HCC progression included HBsAg positivity, a NLR of $>1.85$ and final TACE received, while the embolism volume served as a protective factor. The present study also provides insight into how to reduce the progression of HCC through inhibiting CD151. Whether this approach may be used in the management of HCC is worthy of further study.

\section{References}

1. Torre LA, Bray F, Siegel RL, Ferlay J, Lortet-Tieulent J and Jemal A: Global cancer statistics, 2012. CA Cancer J Clin 65: 87-108, 2015

2. Song P, Tang W, Tamura S, Hasegawa K, Sugawara Y, Dong J and Kokudo N: The management of hepatocellular carcinoma in Asia: A guideline combining quantitative and qualitative evaluation. Biosci Trends 4: 283-287, 2010.

3. Lee KH, Sung KB, Lee DY, Park SJ, Kim KW and Yu JS: Transcatheter arterial chemoembolization for hepatocellular carcinoma: Anatomic and hemodynamic considerations in the hepatic artery and portal vein. Radiographics 22: 1077-1091, 2002.

4. Llovet JM and Bruix J: Systematic review of randomized trials for unresectable hepatocellular carcinoma: Chemoembolization improves survival. Hepatology 37: 429-442, 2003.

5. Marelli L, Stigliano R, Triantos C, Senzolo M, Cholongitas E, Davies N, Tibballs J, Meyer T, Patch DW and Burroughs AK: Transarterial therapy for hepatocellular carcinoma: Which technique is more effective? A systematic review of cohort and randomized studies. Cardiovasc Interv Radiol 30: 6-25, 2007

6. Murata S, Mine T, Ueda T, Nakazawa K, Onozawa S, Yasui D and Kumita S: Transcatheter arterial chemoembolization based on hepatic hemodynamics for hepatocellular carcinoma. ScientificWorldJournal 2013: 479805, 2013.

7. Cheung TT, Poon RT, Jenkins CR, Chu FS, Chok KS, Chan AC, Tsang SH, Dai WC, Yau TC, Chan SC, et al: Survival analysis of high-intensity focused ultrasound therapy vs. transarterial chemoembolization for unresectable hepatocellular carcinomas. Liver Int 34: e136-e143, 2014.

8. Zhong JH and Li LQ: Postoperative adjuvant transarterial chemoembolization for participants with hepatocellular carcinoma: A meta-analysis. Hepatol Res 40: 943-953, 2010.

9. Gao ZH, Bai DS, Jiang GQ and Jin SJ: Review of preoperative transarterial chemoembolization for resectable hepatocellular carcinoma. World J Hepatol 7: 40-43, 2015.

10. Lai EC, Lo CM, Fan ST, Liu CL and Wong J: Postoperative adjuvant chemotherapy after curative resection of hepatocellular carcinoma: A randomized controlled trial. Arch Surg 133: 183-188, 1998.

11. Lin CI, Lin ZY, Hsieh MY, Huang CF, Chen SH and Chuang WL: Potential risk factors for the reactivation of the replication of hepatitis B and C viruses after transcatheter arterial chemoembolization of hepatocellular carcinoma. Kaohsiung J Med Sci 27: 554-559, 2011.

12. Hsieh MY, Lin ZY and Chuang WL: Serial serum VEGF-A, angiopoietin-2, and endostatin measurements in cirrhotic patients with hepatocellular carcinoma treated by transcatheter arterial chemoembolization. Kaohsiung J Med Sci 27: 314-322, 2011

13. Wang Z, Zhou J, Fan J, Tan CJ, Qiu SJ, Yu Y, Huang XW and Tang ZY: Sirolimus inhibits the growth and metastatic progression of hepatocellular carcinoma. J Cancer Res Clin Oncol 135: 715-722, 2009

14. Xiang ZL, Zeng ZC, Fan J, Tang ZY, Zeng HY and Gao DM: Gene expression profiling of fixed tissues identified hypoxia-inducible factor-1 $\alpha$, VEGF, and matrix metalloproteinase-2 as biomarkers of lymph node metastasis in hepatocellular carcinoma. Clin Cancer Res 17: 5463-5472, 2011.

15. Ke AW, Shi GM, Zhou J, Wu FZ, Ding ZB, Hu MY, Xu Y, Song ZJ, Wang ZJ, Wu JC, et al: Role of overexpression of CD151 and/or c-Met in predicting prognosis of hepatocellular carcinoma. Hepatology 49: 491-503, 2009.

16. Kwon MJ, Seo J, Kim YJ, Kwon MJ, Choi JY, Kim TE, Lee DH, Park S, Shin YK, Han J and Choi YL: Prognostic significance of CD151 overexpression in non-small cell lung cancer. Lung Cancer 81: 109-116, 2013.

17. Hashida H, Takabayashi A, Tokuhara T, Hattori N, Taki T, Hasegawa H, Satoh S, Kobayashi N, Yamaoka Y and Miyake M: Clinical significance of transmembrane 4 superfamily in colon cancer. Br J Cancer 89: 158-167, 2003. 
18. Yang YM, Zhang ZW, Liu QM, Sun YF, Yu JR and Xu WX: Overexpression of CD151 predicts prognosis in patients with resected gastric cancer. PLoS One 8: e58990, 2013.

19. Palmer TD, Martínez CH, Vasquez C, Hebron KE, Jones-Paris C, Arnold SA, Chan SM, Chalasani V, Gomez-Lemus JA, Williams AK, et al: Integrin-free tetraspanin CD151 can inhibit tumor cell motility upon clustering and is a clinical indicator of prostate cancer progression. Cancer Res 74: 173-187, 2014.

20. Xu X, Chen W, Zhang L, Miao R, Zhou Y, Wan Y, Dong Y and Liu C: Prognostic significance of neutrophil to lymphocyte ratio in patients with hepatocellular carcinoma after transcatheter arterial chemoembolization. Chin Med J (Engl) 127: 4204-4209, 2014.

21. Li X, Chen ZH, Xing YF, Wang TT, Wu DH, Wen JY, Chen J, Lin Q, Dong M, Wei L, et al: Platelet-to-lymphocyte ratio acts as a prognostic factor for patients with advanced hepatocellular carcinoma. Tumor Biol 36: 2263-2269, 2015

22. Lee $\mathrm{CH}$, Lin YJ, Lin CC, Yen CL, Shen CH, Chang CJ and Hsieh SY: Pretreatment platelet count early predicts extrahepatic metastasis of human hepatoma. Liver Int 35: 2327-2336, 2015.

23. Pugh RN, Murray-Lyon IM, Dawson JL, Pietroni MC and Williams R: Transection of the oesophagus for bleeding oesophageal varices. Br J Surg 60: 646-649, 1973.

24. Shuster A, Huynh TJ, Rajan DK, Marquez MA, Grant DR, Huynh DC and Jaskolka JD: Response evaluation criteria in solid tumors (RECIST) criteria are superior to European Association for Study of the Liver (EASL) criteria at 1 month follow-up for predicting long-term survival in patients treated with transarterial chemoembolization before liver transplantation for hepatocellular cancer. J Vasc Interv Radiol 24: 805-812, 2013.

25. Ashman LK, Aylett GW, Mehrabani PA, Bendall LJ, Niutta S Cambareri AC, Cole SR and Berndt MC: The murine monoclonal antibody, 14A2.H1, identifies a novel platelet surface antigen. $\mathrm{Br}$ J Haematol 79: 263-270, 1991.

26. Hasegawa H, Watanabe $H$, Nomura $T$, Utsunomiya $Y$, Yanagisawa $\mathrm{K}$ and Fujita S: Molecular cloning and expression of mouse homologue of SFA-1/PETA-3 (CD151), a member of the transmembrane 4 superfamily. Biochim Biophys Acta 1353: $125-130,1997$
27. Hasegawa $\mathrm{H}$, Kishimoto $\mathrm{K}$, Yanagisawa $\mathrm{K}$, Terasaki $\mathrm{H}$, Shimadzu M and Fujita S: Assignment of SFA-1 (PETA-3), a member of the transmembrane 4 superfamily, to human chromosome $11 \mathrm{p} 15.5$ by fluorescence in situ hybridization. Genomics 40 : 193-196, 1997.

28. Nicole Johnson and Susan B. Wormsley: Human leukocyte differentiation antigens of the sixth international workshop: What can we expect to see in the clinic? Clinical Immunology Newsletter 18: 33-42, 1998.

29. Sincock PM, Mayrhofer G and Ashman LK: Localization of the transmembrane 4 superfamily (TM4SF) member PETA-3 (CD151) in normal human tissues: Comparison with CD9, CD63, and alpha5betal integrin. J Histochem Cytochem 45: 515-525, 1997.

30. Sadej R, Grudowska A, Turczyk L, Kordek R and Romanska HM: CD151 in cancer progression and metastasis: A complex scenario. Lab Invest 94: 41-51, 2014.

31. Zhu M, Guo J, Xia H, Li W, Lu Y, Dong X, Chen Y, Xie X, Fu S and Li M: Alpha-fetoprotein activates AKT/mTOR signaling to promote CXCR4 expression and migration of hepatoma cells. Oncoscience 2: 59-70, 2015.

32. Shi GM, Ke AW, Zhou J, Wang XY, Xu Y, Ding ZB, Devbhandari RP, Huang XY, Qiu SJ, Shi YH, et al: CD151 modulates expression of matrix metalloproteinase 9 and promotes neoangiogenesis and progression of hepatocellular carcinoma. Hepatology 52: 183-196, 2010.

33. Fei Y, Wang J, Liu W, Zuo H, Qin J, Wang D, Zeng H and Liu Z: CD151 promotes cancer cell metastasis via integrins $\alpha 3 \beta 1$ and a6ß1 in vitro. Mol Med Rep 6: 1226-1230, 2012.

34. Ke AW, Shi GM, Zhou J, Huang XY, Shi YH, Ding ZB, Wang XY, Devbhandari RP and Fan J: CD151 amplifies signaling by integrin $\alpha 6 \beta 1$ to PI3K and induces the epithelial-mesenchymal transition in HCC cells. Gastroenterology 140: 1629-1641.e15, 2011.

35. Jia ZZ, Jiang GM and Feng YL: Serum HIF-1alpha and VEGF levels pre- and post-TACE in patients with primary liver cancer. Chin Med Sci J 26: 158-162, 2011

36. Cillo U, Vitale A, Grigoletto F, Farinati F, Brolese A, Zanus G, Neri D, Boccagni P, Srsen N, D'Amico F, et al: Prospective validation of the Barcelona clinic liver cancer staging system. J Hepatol 44: 723-731, 2006. 